\title{
ORIGINAL
}

\section{EXPOSICIÓN LABORAL A MYCOBACTERIUM BOVIS MULTIRRESISTENTE EN UN HOSPITAL DE ZARAGOZA}

Víctor Manuel Solano Bernad, María Jesús Hernández Navarrete, Juan Ignacio Martín Sánchez, Elena Vanessa Martínez Sánchez y José Luis Arribas Llorente

Servicio de Medicina Preventiva. Hospital Universitario Miguel Servet, Zaragoza

\section{RESUMEN}

Fundamento: Los trabajadores del medio hospitalario están expuestos a diversos riesgos laborales, aunque los más específicos son los biológicos. Dentro de ellos, los asociados a la transmisión respiratoria y en concreto a la tuberculosis, ocupan un lugar destacaClobjetivo de este traba tados de un protocolo de evaluación y vigilancia tras un exposición laboral a Mycobacterium bovis multiresistente (MbMR).

Método: Un paciente varón fue diagnosticado en 1999 de infección por MbMR tras 10 días sin aislamiento respiratorio, en el hospital Miguel Servet (Zaragoza). Durante ese tiempo estuvo en contacto con 167 trabajadores de distintos servicios hospitalarios. Se elaboró un protocolo de vigilancia y control de contactos basado en: cumplimentación de una encuesta y realización de un Mantoux inicial (si el trabajador era tuberculín negativo previo) y tres meses después, radiografía de tórax y seguimiento clínico de 2 años (controles cada 3 meses) en tuberculín positivos y la no administración de quimioprofilaxis aunque se evidenciara infección.

Resultados: Se obtuvo información de 160 trabajadores (96\%). 94 trabajadores (59\%) tenían realizado un Mantoux previo y 7 habían padecido tuberculosis. Fue necesario el seguimiento de 61 tuberculín positivos (29 previamente positivos y 32 detectados en el Mantoux inicial). Ningún trabajador con Mantoux inicial negativo tuvo un resultado positivo al repetirlo a los 3 meses ni manifestó síntomas sugerentes de transmisión durante el período de seguimiento. Algunas variables, como la edad o trabajar en el servicio de Infecciosas, se asociaron de forma estadísticamente significativa con la necesidad de seguimiento.

Conclusiones: El riesgo de transmisión ocupacional tras un contacto con MbMR podría ser similar a $M$. tuberculosis, aunque es necesaria mayor experiencia para confirmar este hecho. Es importante un diagnóstico precoz y la existencia de un protocolo para implementar medidas preventivas y de control en este tipo de contactos laborales.

Palabras clave: Enfermedades infecciosas y parasitarias. Tuberculosis bovina. Riesgos laborales. Salud laboral. Personal sanitario. Síndrome de inmunodeficiencia adquirida. Tuberculosis.

\section{Correspondencia:}

José Luis Arribas Llorente

Servicio de Medicina Preventiva

Hospital Universitario Miguel Servet

Paseo Isabel la Católica 1-3

50009 Zaragoza

976765557

Fax: 976566234

Correo electrónico: jarribas@hmservet.insalud.es

\section{ABSTRACT}

\section{Occupational Exposure to Multiresistant Mycobacterium Bovis at a Hospital in Zaragoza, Spain}

Background: Those working in a hospital environment are exposed to different occupational risks, although more specifically biological ones. One of the major risks is that of exposure by airborne transmission, more specifically, tuberculosis. This study is aimed at providing a description and analysis of the results of the implementation of an evaluation and surveillance protocol following occupational exposure to Multiresistant mycobacterium bovis (MRMb).

Method: A male patient was diagnosed with MRMb infection at the Miguel Servet Hospital in Zaragoza in 1999 following ten days without respiratory isolation. During this period, he came into contact with 167 employees from different hospital departments. A surveillance and contact control protocol was prepared based on: completing a survey and undergoing an initial Mantoux (if the employee had previously tested negative for tuberculin), followed three months later by a chest X-ray and then a two-year clinical follow-up (check-ups every three months) for those having tested positive for tuberculin and no administering of chemoprophylaxis even though signs of infection were to have been found.

Results: Information was gathered on 160 employees (96\%). A total of 94 employees (59\%) had previously undergone a Mantoux, seven (7) having had tuberculosis. It was necessary for a follow-up to be conducted on sixty-one (61) employees who tested positive ( 29 previously positive and 32 detected in the initial Mantoux). No employee who had tested negative on an initial Mantoux tested positive on repeated testing three months later nor showed any symptoms indicative of transmission during the follow-up period Some variables, such as age or working in the Infectious Disease Unit were related, on a statistically significant basis, to follow-up being required.

Conclusions: The risk of occupational transmission following contact with MRMb might be similar to M. Tuberculosis, although further experience would be required in order to confirm this fact. Early diagnosis and availing of a protocol for implementing measures aimed at preventing and controlling this type of occupational exposure are of importance.

Key words:. Communicable diseases. Occupational hazards. Occupational health. Healthcare personnel. Acquired immunodeficiency syndrome. Tuberculosis. 


\section{INTRODUCCIÓN}

El principal agente etiológico de la tuberculosis (TBC) humana es Mycobacterium tuberculosis, pero existe una proporción de casos desconocida debida a Mycobacterium bovis (agente productor de la TBC bovina). Se estima que entre 1954 y 1970 la proporción de casos en seres humanos debidos a $M$. bovis en todo el mundo fue de un 3,1\% respecto a todas las formas de TBC, siendo el $2,1 \%$ de las formas pulmonares y el 9,4\% de las formas extrapulmonares ${ }^{1}$. La TBC causada por M. bovis es clínicamente indistinguible de la causada por M. tuberculosis.

Respecto a la TBC, España se sitúa en el ámbito europeo, en cuanto a tasas de incidencia, en segundo lugar tras Portugal, según la Red de Vigilancia Europea de Tuberculosis (Euro TB) ${ }^{2}$. Todo ello a pesar de observarse en los últimos años un descenso paulatino de sus tasas de incidencia, aunque en nuestro país sólo son de declaración obligatoria la TBC respiratoria y la TBC meníngea. La alta incidencia de TBC en el grupo de edad entre los 25-44 años podría explicarse en parte teniendo en cuenta el hecho de que España se encuentra detrás de Portugal en cuanto a tasas de incidencia de sida y que el riesgo de padecer TBC depende del estado inmunitario de cada individuo. El aumento del número de personas inmigrantes procedentes de países con altas tasas de incidencia de $\mathrm{TBC}$, podría ser otro factor a tener en cuenta. Tampoco hay que olvidar los brotes de TBC que siguen apareciendo en distintas instituciones en las que las condiciones de hacinamiento favorecen la diseminación de la micobacteria (guarderías, colegios y residencias de la tercera edad $)^{2}$.

En aquellos países donde la TBC bovina no está controlada, la mayoría de los casos de TBC tienen lugar en personas jóvenes a causa de la ingesta de leche o por la manipulación de la misma, con clínica no pulmonar. A su vez, los agricultores pueden adquirir la infección por vía inhalatoria a partir del ganado infectado, desarrollando la forma típica pulmonar. En los países desarrollados, el control y eliminación de la TBC en animales, junto con la pasteurización de la leche ha reducido la incidencia de la enfermedad causada por M. bovis tanto en el ganado vacuno como en los seres humanos, pudiendo aparecer casos en personas ancianas como consecuencia de la reactivación del bacilo ${ }^{1}$.

En los países industrializados de nuestro entorno se ha aislado $M$. bovis en personas infectados por el HIV $\mathrm{HI}^{3-4}$. En Francia, la infección por $M$. bovis es del $1,6 \%$ en pacientes HIV positivos, en los que todas las muestras aisladas eran resistentes a la isoniazida, concluyéndose que $M$. bovis presenta una resistencia natural a la pirazinamida ${ }^{5}$. En nuestro país se han producido brotes nosocomiales de $M$. bovis multiresistente (MbMR) desde 1991, cuando comenzó el primer brote en un hospital de Madrid ${ }^{6}$. Posteriormente, la epidemia se extendió a otros hospitales de Madrid, Málaga, Cádiz y Valencia, acumulándose un total de 111 casos en 5 años ${ }^{7-10}$.

En nuestro medio las cepas resistentes de Mycobacterium tuberculosis oscilan entre un 2,7 y un $7,5 \%$, y las cepas multiresistentes entre un 0 y un $1,9 \%^{11}$. Diversos hechos están jugando un papel importante en la aparición de resistencias a los antituberculosos de primera línea, que se da sobre todo en personas jóvenes y se asocia a la coinfección por $\mathrm{VIH}^{12}$. La existencia de una vigilancia de estas cepas multiresistentes puede facilitar el control de nuevos brotes, evitando que puedan convertirse en graves problemas de Salud Pública.

Los trabajadores del medio hospitalario están expuestos a diversos riesgos laborales, aunque los más específicos son los biológicos. Dentro de ellos, los asociados a la transmisión respiratoria, en concreto a la TBC, ocupan un lugar destacado. La TBC multiresistente en individuos inmunodeprimidos añade una nueva dimensión al problema, porque a pesar del avance en las medidas de 
prevención, existen carencias en la conciencia del riesgo por parte del personal.

En marzo de 1999, un varón de 50 años ingresó en el Hospital Universitario Miguel Servet (Zaragoza) y tras la realización de una baciloscopia y cultivo y estudio genotípico posterior de un esputo inducido se identificó la presencia de MbMR.

El objetivo de este trabajo es describir y analizar los resultados de un protocolo de evaluación y vigilancia implantado tras esta exposición laboral hospitalaria con un paciente infectado por MbMR.

\section{SUJETOS Y MÉTODO}

El paciente estaba diagnosticado de esclerodermia tratada con dosis bajas de corticoides. Acudió a urgencias refiriendo cansancio y febrícula (pero no tos) de una semana de evolución. La radiografía de tórax fue normal y se le diagnosticó una reagudización de su proceso de esclerodermia, siendo ingresado en hospital de día. Durante los 10 días de estancia en esta unidad no manifestó mejoría clínica y una imagen radiológica no concluyente motivó la realización de la baciloscopia. Ante un resultado sugestivo de la presencia de micobacterias, se le practicó un esputo inducido y se le situó en aislamiento respiratorio en la Unidad de Infecciosos. Durante su estancia en esta Unidad empeoró clínicamente hasta la situación de insuficiencia respiratoria aguda. Por falta de camas en la Unidad de Cuidados Intensivos fue trasladado a otro hospital de nuestra ciudad donde posteriormente falleció.

El cultivo y estudio genotípico posterior del esputo inducido mediante RFLP (restriction fragment length polymorphism) y spoligotyping identificó la presencia de M. bovis resistente a isoniazida, estreptomicina, rifampicina, ácido aminoparasalicílico y etambutol. La comparación del patrón mostrado con estas técnicas fue idéntico al pre- sentado por otras cepas aisladas responsables del brote nosocomial descrito en nuestro país.

Durante los 10 días de hospitalización durante los que el paciente estuvo sin aislamiento, se estimó que de los 5.000 trabajadores del hospital 167 pudieron tener contacto con el paciente o con muestras biológicas de $M$. bovis. Dado el intervalo de tiempo transcurrido entre la posible exposición y la recepción de los resultados del cultivo no se pudieron fijar criterios espacio-temporales precisos para definir o categorizar la exposición. Se consideró como potencialmente expuesto a cualquier trabajador que hubiera tenido contacto, aunque fuera esporádico, con el paciente. Estos trabajadores pertenecían fundamentalmente a los servicios de Urgencias, Hospital de Día (Unidad de agudos), Unidad de Infecciosos y Microbiología (Unidad de micobacterias). Se incluyó a este último personal ante la posibilidad de un incumplimiento de las normas de prevención en el uso de los equipos de protección individual y de las cabinas de bioseguridad.

Ante la situación descrita, los técnicos de salud pública elaboraron un protocolo de vigilancia y control que posteriormente fue consensuado con los responsables de Medicina Preventiva de los hospitales implicados. Los puntos más destacables respecto a la identificación y estudio de contactos (personal potencialmente expuesto) fueron los siguientes: 1) Evaluación inicial mediante entrevista y cumplimentación de una encuesta, con especial atención en la valoración de factores subyacentes que favorecieran la infección y desarrollo de la enfermedad tuberculosa. 2) Realización de una prueba de Mantoux inicial y repetición de la misma a los tres meses en caso de negatividad de la primera. Si la prueba seguía siendo negativa, se consideraba finalizado el seguimiento del trabajador. Se consideró como positivo un resultado superior a 5 milímetros de induración en trabajadores no vacunados frente a TBC y superior a $14 \mathrm{~mm}$ en los suje- 
tos previamente vacunados. Se tuvo en cuenta el efecto Booster repitiendo la prueba de Mantoux con un intervalo mínimo de una semana en los vacunados, con resultado negativo. 3) Realización de una radiografía de tórax más baciloscopia a todo trabajador con resultado positivo del Mantoux.

En los trabajadores tuberculín positivos (ya conocidos previamente a la aplicación del protocolo o detectados con la prueba de Mantoux inicial) se realizó un seguimiento clínico cada tres meses durante dos años mediante entrevista clínica. La realización de pruebas quedaba a criterio del facultativo en función de las circunstancias individuales de cada caso. No se administró quimioprofilaxis con isoniazida a los trabajadores con Mantoux positivo dada la resistencia de MbMR a este fármaco. Todas las notificaciones de citación a cada una de las personas en seguimiento se realizaron mediante carta certificada.

Las principales variables incluidas en la encuesta y analizadas fueron estructuradas en distintos apartados:

- Datos personales: edad, sexo y puesto de trabajo.

- Antecedentes: vacunación previa y fecha, historia anterior de TBC, fecha de realización y resultado de pruebas de Mantoux previas (en cualquier momento), resultados de radiografías de tórax o baciloscopias previas (por estudios de contactos previos), tratamiento anterior con isoniacida (quimioprofilaxis secundaria) y contacto previo con pacientes enfermos de TBC (hospitalario y/o familiar).

- Presencia de enfermedades o factores subyacentes: virus de la inmunodeficiencia humana, usuario de drogas por vía parenteral, diabetes, tratamiento inmunosupresor, insuficiencia renal, leucemias/linfomas, tumores sólidos, enfermedad pulmonar obstructiva crónica (EPOC), silicosis, hepatopatías, alteraciones digestivas.

- Valoración del riesgo de contacto: área de trabajo y medidas de prevención utilizadas (aislamiento estricto, uso de respirador, bata y guantes).

- Prueba de Mantoux inicial: fecha de realización y resultado del Mantoux basal, fecha de realización y resultado del efecto Booster (cuando fue necesario), resultados de radiografías de tórax y baciloscopias (cuando se solicitaron).

- Seguimiento de la prueba de Mantoux: fecha de realización y resultado del Mantoux realizado a los tres meses, resultados de radiografías de tórax y baciloscopias.

El análisis estadístico descriptivo de los resultados se realizó por medio del cálculo de proporciones y medias aritméticas y de sus intervalos de confianza al 95\% (IC95\%). El estudio analítico se realizó mediante las pruebas estadísticas de la Chi-cuadrado o el test exacto de Fisher en el contraste de variables cualitativas y la t de Student cuando se trataba de contrastar el valor de una variable cuantitativa, según la distribución de una cualitativa binaria. Se consideró una diferencia como estadísticamente significativa cuando el valor asociado de $\mathrm{p}$ era inferior a 0,05 . Se utilizaron los programas estadísticos SPSS (versión 8.0, SPSS, Chicago, IL) y Epi-info (versión 6, CDC, Atlanta).

\section{RESULTADOS}

La distribución por profesiones de los 167 trabajadores calificados como expuestos fue la siguiente: el $42 \%$ fueron diplomadas de enfermería (DUE), el 35\% auxiliares de enfermería (AE), el 11\% celadores, el 9\% médicos y un $3 \%$ personal de limpieza y otros. Por unidades, un $45 \%$ del personal 
Figura 1

Descripción del proceso seguido por los trabajadores incluidos en el protocolo
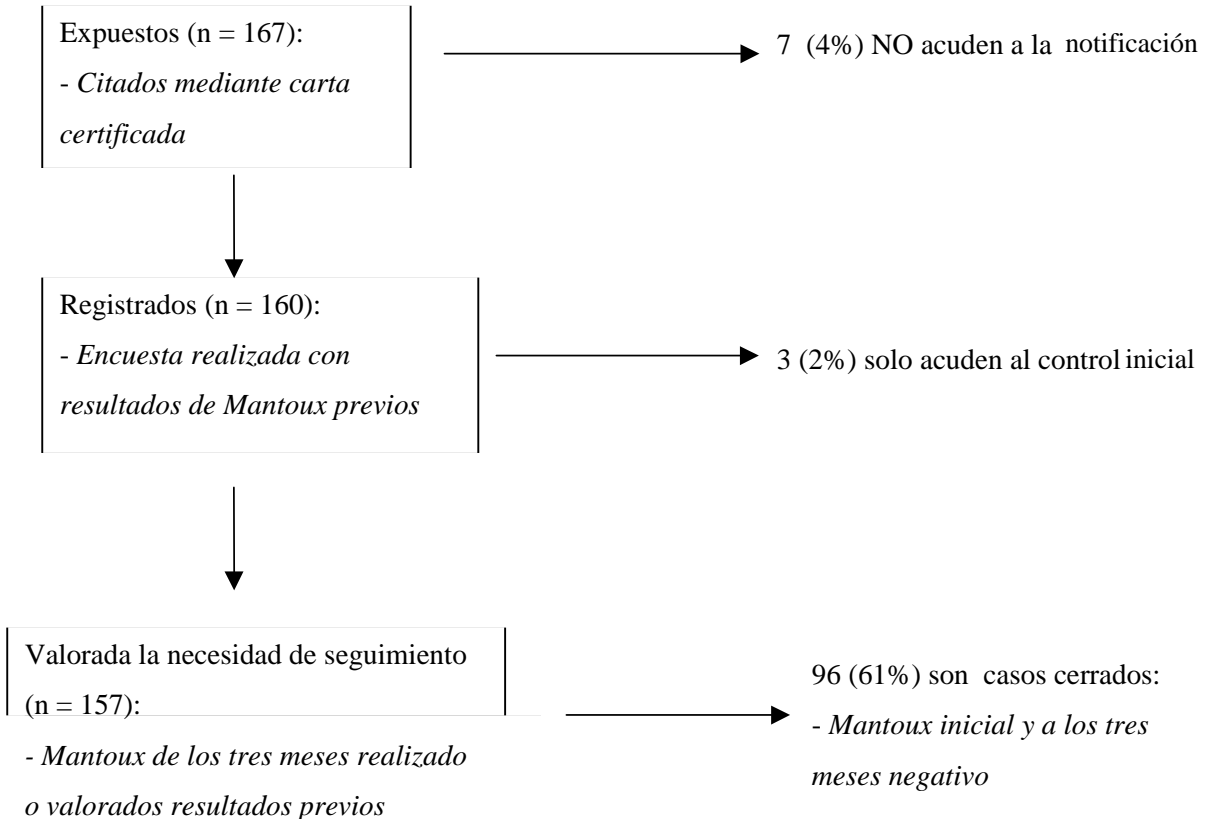

$61(39 \%)$ es necesario el seguimiento:

- Mantoux previo (en cualquier

momento) positivo $(n=29)$ ó

- Mantoux inicial positivo $(n=32)$

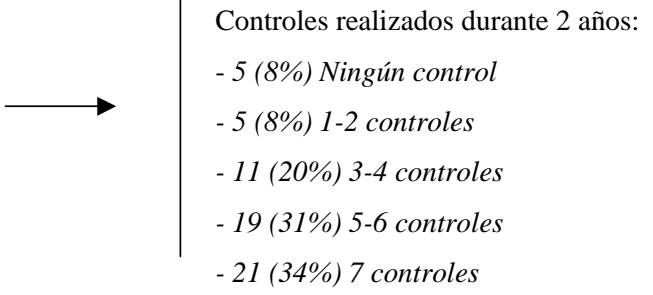

pertenecía a la unidad de agudos, un $27 \%$ a urgencias, un $17 \%$ a infecciosos, un $9 \%$ a microbiología y un $2 \%$ a otras (ambulancia).

años (IC95\%: 41,21-44,09). En la tabla 1 se presenta la descripción de las principales variables recogidas en el estudio. El 50\% de los trabajadores estaban vacunados frente a la TBC y siete trabajadores habían sido diagnosticados y tratados con anterioridad frente a esta enfermedad. De estos trabajadores que habían padecido TBC, el 86\% manifestaron bajadores hospitalarios (figura 1). Un $84 \%$ eran mujeres y la edad media fue de 42,65 
Tabla 1

Descripción de las variables incluidas en la encuesta $(n=160)$

\begin{tabular}{|c|c|c|}
\hline \multirow[t]{2}{*}{ Género } & Hombres & $25(15,6 \%)$ \\
\hline & Mujeres & $135(84,4 \%)$ \\
\hline Edad Media & & 42,65 años (IC95\%: 41,21 - 44,09) \\
\hline BCG previa Sujetos vacunados anteriormente & & $80(50 \%)(\mathrm{IC} 95 \%: 42-58)$ \\
\hline $\mathrm{TBC}$ anterior & & $7(4,4 \%)(\mathrm{IC} 95 \%: 1,8-8,8)$ \\
\hline Mantoux previo & & $94(58,8 \%)(\mathrm{IC} 95 \%: 50,7-66,5)$ \\
\hline Radiografía de tórax previa & & $35(21,9 \%)($ IC95\%: 15,7-29,1) \\
\hline Baciloscopia previa & & $6(3,8 \%)(\mathrm{IC} 95 \%: 1,4-8)$ \\
\hline Tratamiento previo con isoniacida & & $15(9,4 \%)(\mathrm{IC} 95 \%: 5,3-15)$ \\
\hline Antecedentes familiares de tuberculosis & & $15(9,4 \%)(\mathrm{IC} 95 \%: 5,3-15)$ \\
\hline Radiografia de tórax a causa del protocolo & & $55(34,4 \%)(\mathrm{IC} 95 \%: 27,1-42,3)$ \\
\hline
\end{tabular}

haber tenido otros contactos no hospitalarios y el $43 \%$ presentaban otros factores de riesgo como diabetes mellitus, EPOC y alteraciones digestivas. El resto de trabajadores no comunicaron factores de riesgo clínico relevantes.

El 59\% de los trabajadores se habían realizado la prueba del Mantoux con anterioridad a la aplicación de este protocolo por diversos motivos, siendo positiva en 29 de ellos (31\%). De las 131 pruebas de Mantoux realizadas en este estudio (a los trabajadores con resultado previo negativo o que nunca se la habían realizado) tuvieron un resultado positivo $32(25 \%)$. Esto suponía que el 19\% de los trabajadores previamente tuberculínnegativos fueron positivos a la prueba del Mantoux en esta consulta inicial, sin poder determinar en qué momento del pasado se produjo la seroconversión. En ningún trabajador con Mantoux inicial negativo se demostró un resultado positivo al repetirlo a los 3 meses. En tres trabajadores no se pudo determinar específicamente la necesidad de seguimiento al no haber acudido al control de los 3 meses (figura 1).

Por tanto, tras la realización de la prueba de Mantoux inicial y su repetición a los tres meses, se determinó finalmente realizar el seguimiento de 61 trabajadores (29 tubercu- lín positivos ya conocidos y 32 detectados en el control inicial) para valorar si desarrollaban manifestaciones clínicas de enfermedad.

De este grupo de trabajadores en seguimiento, el 34\% acudió a todos los controles realizados cada tres meses hasta completar el período de seguimiento propuesto de dos años. Ningún trabajador manifestó signos y/o síntomas sugerentes de transmisión y desarrollo de la enfermedad (astenia, pérdida de apetito, febrícula vespertina...) en las entrevistas realizadas durante este tiempo. En la actualidad tampoco se ha constatado la existencia de casos de infección secundaria a través de otras fuentes de información (laboratorio de microbiología, autoridades de salud pública, etc...).

Los resultados más relevantes de la comparación de las características entre los trabajadores que precisaron seguimiento (tuberculín positivos) y los que no lo precisaron (tuberculín negativos) se presentan en la tabla 2. Se encontraron diferencias estadísticamente significativas entre trabajar en el servicio de Enfermedades Infecciosas y la necesidad de realizar el seguimiento $(\mathrm{p}<0,05)$, al igual que en aquellos sujetos diagnosticados y tratados frente a la TBC anteriormente $(\mathrm{p}<0,01)$, así como tener realizada previamente una radiografía de tórax, 
Tabla 2

Descripción de las características de los trabajadores según la positividad del Mantoux (n = 157)

\begin{tabular}{|c|c|c|c|}
\hline & Mantoux positivo & Mantoux negativo & \\
\hline & $(n=61)$ & $(n=96)$ & \\
\hline \multirow[t]{2}{*}{ Género } & Hombres: $10(16,4 \%)$ & Hombres: $14(14,6 \%)$ & 0,82 \\
\hline & Mujeres: $51(83,6 \%)$ & Mujeres: $82(85,4 \%)$ & \\
\hline Edad media & 44,7 años (IC95\%: 42,6 - 46,8) & 41,7 años (IC95\%: 39,7 - 43,6) & 0,044 \\
\hline \multirow[t]{5}{*}{ Estamento } & Médico: $7(11,5 \%)$ & Médico: $5(5 \%)$ & 0,54 \\
\hline & DUE: $25(41 \%)$ & DUE: $42(44 \%)$ & \\
\hline & AE: $22(36 \%)$ & AE: $34(35 \%)$ & \\
\hline & Celador: $5(8 \%)$ & Celador: $13(14 \%)$ & \\
\hline & Otros: $2(3 \%)$ & Otros: $2(2 \%)$ & \\
\hline \multirow[t]{5}{*}{ Servicio/Unidad } & Agudos: $26(43 \%)$ & Agudos: $45(47 \%)$ & 0,23 \\
\hline & Infecciosos: $16(26 \%)$ & Infecciosos: $12(13 \%)$ & \\
\hline & Urgencias: $13(21 \%)$ & Urgencias: $30(32 \%)$ & \\
\hline & Microbiología: $5(8 \%)$ & Microbiología: 7 (7\%) & \\
\hline & Otras: $1(2 \%)$ & Otras: $1(1 \%)$ & \\
\hline BCG previa & $32(52,5 \%)(\mathrm{IC} 95 \%: 39,3-65,4)$ & $47(49 \%)($ IC95\%: 38,6-59,4) & 0,74 \\
\hline $\mathrm{TBC}$ anterior & 7 (11,5\%) (IC95\%: 4,7-22,2) & $0(0 \%)$ & 0,001 \\
\hline Mantoux previo & $40(65,6 \%)(\mathrm{IC} 95 \%: 52,3-77,3)$ & $54(56,3 \%)(\mathrm{IC} 95 \%: 45,7-66,4)$ & 0,32 \\
\hline Radiografía previa tórax & $26(42,6 \%)$ (IC95\%: 30-55,9) & $9(9,4 \%)($ IC95\%: 4,4-17) & $<0,001$ \\
\hline BK previa & $6(10 \%)(\mathrm{IC} 95 \%: 3,7-20,2)$ & $0(0 \%)$ & 0,003 \\
\hline Tratamiento previo & $14(23 \%)($ IC95\%: 13,2-35,5) & $1(1 \%)(\mathrm{IC} 95 \%: 0-5,7)$ & $<0,001$ \\
\hline FR familiar & $7(11,5 \%)(\mathrm{IC} 95 \%: 4,7-21,4)$ & $8(8,3 \%)($ IC95\%: 3,9-15,2) & 0,58 \\
\hline
\end{tabular}

Los resultados se expresan en números absolutos, medias y porcentajes con su correspondiente IC95\%.

DUE: Diplomada Universitaria en Enfermería. AE: Auxiliar de Enfermería. BCG previa: Sujetos vacunados anteriormente. TBC anterior: Sujetos anteriormente diagnosticados y tratados frente a la TBC. Mantoux previo: Sujetos a los que se les había realizado previamente al estudio (por estudios de contactos realizados en cualquier momento) una prueba de Mantoux. RxTx previa: Sujetos a los que se les había realizado previamente al estudio (por estudios de contactos realizados en cualquier momento) una radiografía de tórax. BK previa: Sujetos a los que se les había realizado previamente al estudio una baciloscopia. Tto previo: Sujetos que habían sido tratados con isoniacida previamente al estudio (por quimioprofilaxis secundaria). FR familiar: Sujetos que tuvieron contacto previo con la TBC en el ámbito familiar.

baciloscopia y la administración de isoniazida $(\mathrm{p}<0.01)$. Se observaron diferencias estadísticamente significativas según el Mantoux previo fuese positivo o negativo (en cualquier momento) y la necesidad de seguimiento $(\mathrm{p}<0,01)$. Los sujetos en seguimiento tenían una edad media de 44,7 años, superior en 3 años a la de los que no lo precisaron $(p<0,05)$. No se encontraron diferencias estadísticamente significativas entre la vacunación previa con BCG y la necesidad de realizar el seguimiento (por ser tuberculín positivos en cualquier momento), que se realizó en el 40,5\% de los vacunados y en el $37 \%$ de los no vacunados. No se encontró ningún resultado destacable del análisis de las medidas de prevención utilizadas.

\section{DISCUSIÓN}

Los datos de transmisión nosocomial y comunitaria de $M$. bovis en España, Francia e Italia con transmisión persona-persona que conduce a enfermedad activa, el corto intervalo de tiempo infección-enfermedad en sujetos inmunodeprimidos, y la potencial diseminación de cepas de MbMR, remarcan la importancia de identificar con rapidez los posibles casos secundarios de infección por 
este microorganismo. Estos datos confirman la necesidad de destinar los recursos necesarios para el seguimiento de contactos con personas infectadas, puesto que pueden constituir un grave problema de salud pública.

El caso fuente que originó este estudio de contactos estaba infectado por la misma cepa productora de los últimos brotes nosocomiales descritos en nuestro país, así como otra persona fallecida en nuestro hospital en 1996 que también produjo un caso aislado, y confirma la letalidad de la misma ${ }^{6-10,13}$. El pronóstico de estos sujetos, especialmente los coinfectados por MbMR/VIH, es generalmente muy desfavorable, aunque existe documentación sobre diferentes posibilidades terapéuticas ${ }^{14}$.

Respecto al seguimiento de estos contactos laborales, debemos destacar que no se registrara ninguna infección secundaria. En ninguno de los trabajadores estudiados se produjo un viraje en la prueba del Mantoux, esto es, ninguno pasó de ser negativo en el control inicial a positivo en el control de los tres meses. Tampoco se detectó ningún signo o síntoma de enfermedad clínica. Esto podría indicar una transmisibilidad similar a $M$. tuberculosis, aunque es evidente que se necesita una mayor experiencia y número de estudios para valorar esta afirmación. El cumplimiento del seguimiento de nuestros trabajadores no ha sido especialmente remarcable (solo un 34\% del personal acudió a todas las consultas), aunque debemos valorar el largo período de seguimiento con varias consultas y que el $96 \%$ de los trabajadores potencialmente expuestos acudieron al menos a una visita, cifra similar a la registrada en otros estudios ${ }^{6}$.

La realización de una prueba de Mantoux en la primera consulta y su repetición a los tres meses podría ser insuficiente, puesto que se han descrito casos de sujetos inmunocompetentes con prueba negativa de la tuberculina ${ }^{15}$. Aunque no se pudo recoger información de todos los sujetos que tuvie- ron contacto con el enfermo, a todos los trabajadores que acudieron a la primera consulta se les informó y aclaró cualquier duda sobre los signos y los síntomas que pueden aparecer asociados a la infección o al desarrollo de la enfermedad. En este sentido, asumimos que cualquier trabajador afectado (hubiera acudido o no a la primera consulta) habría contactado de alguna forma con el sistema sanitario y se habría tenido constancia de ello por otras fuentes de información relacionadas con la Salud Pública.

Durante el periodo de estudio solo se recogió información sobre la aparición de manifestaciones clínicas de enfermedad. El protocolo no recogía la posibilidad de realizar ninguna prueba de Mantoux después de los tres meses, porque solo entraron en seguimiento aquellos trabajadores con resultado positivo. En los sujetos con resultado negativo en la primera visita y la posterior a los tres meses se estipuló que había transcurrido el tiempo suficiente como para descartar una conversión futura tras el contacto con el paciente.

Como resumen final, nos gustaría insistir en que dado el carácter de multirresistencia de este germen, la alta mortalidad asociada y la posibilidad de transmisión entre hospitales y a los individuos de la comunidad, es importante una diagnóstico precoz de los casos, una correcta identificación de la cepa con su patrón de resistencias, y un estudio de los contactos para la implementación de medidas de control y preventivas como las descritas.

\section{BIBLIOGRAFÍA}

1. Cosivi O, Grange JM, Daborn CJ, Raviglione MC, Fujikura T, Cousins D et al. Zoonotic tuberculosis due to Mycobacterium bovis in developing countries. Emerg Infect Dis 1998; 4 (1): 59-70.

2. Rodríguez Valín E. Situación actual de la tuberculosis en España: Incidencia y mortalidad desde 1995. Características de los casos de tuberculosis y meningitis tuberculosa declarados en 2000. Bol Epidemiol Semanal 2001; 9 (28): 293-6. 
3. Bouvet E, Casalino E, Mendoza-Sassi G, Lariven $\mathrm{S}$, Vallee E, Pernet M et al. A nosocomial outbreak of multidrug-resistant Mycobacterium bovis among HV-infected patients. A case-control study. AIDS 1993; 7:1453-60.

4. Schultsz C, Kuijper EJ, van Soolingen D, Prins JM. Disseminated infection due to multidrug-resistant Mycobacterium bovis in a patient who was seropositive for human inmunodeficiency virus. Clin Infect Dis 1996; 23:841-3.

5. Somoskovi A, Parsons LM, Salfinger M. The molecular basis of resistance to isoniazid, rifampin and pyrazinamide in Mycobacterium tuberculosis. Respir Res 2001; 2: 164-8.

6. Rullán JV, Herrera D, Cano R, Moreno V, Godoy P, Peiró EF et al. Nosocomial transmission of multidrug-resistant Mycobacterium tuberculosis in Spain. Emerg Infect Dis 1996; 2:125-9.

7. Guerrero A, Cobo J, Fortún J, Navas E, Quereda C, Asensio A, et al. Nosocomial transmission of Mycobacterium bovis resistant to 11 drugs in people with advanced HIV-1 infection. Lancet 1997; 350: 1738-42.

8. Samper S, Martín C, Pinedo A, Rivero A, Blázquez J, Baquero F, et al. Transmisión between HIVinfected patients of multidrug-resistant tuberculosis caused by Mycobacterium bovis. AIDS 1997; 11: $1237-42$

9. Rivero A, Márquez M, Santos J, Pinedo A, Sánchez MA, Esteve A et al. High rate of tuberculosis reinfection during a nosocomial outbreak of mul- tidrug-resistant tuberculosis caused by Mycobacterium bovis strain B. Clin Infect Dis 2001; 32:5961.

10. Palenque E, Villena V, Rebollo MJ, Jiménez MS, Samper S. Transmission of multidrug-resistant Mycobacterium bovis to an inmunocompetent patient. Clin Infect Dis 1998; 26:995-6.

11. Idígoras P, Valiente A, Iglesias L, Piñeiro L, PérezTrallero E. Bajo nivel de resistencia a fármacos en cepas de Mycobacterium tuberculosis aisladas en Guipuzkoa (1993-2001). Enferm Infecc Microbiol Clin 2002; 20(4): 187-90.

12. Pablos-Méndez A, Raviglione MC, Laszlo A, Binkin N, Rieder HL, Bustreo F et al. Global surveillance for antituberculosis-drug resistance, 19941997. N Engl J Med 1998: 338 (23): 1641-9.

13. Hermida I, Lezcano MA, García F, Ramos C, Martín C, Aguirre JM. Tuberculosis multirresistente por M. Bovis en paciente con el virus de la inmunodeficiencia humana. Rev Clin Esp 1998; 198(4): 261-2.

14. Valencia ME, Moreno V, Laguna y Juan F, González-Lahoz M. Tuberculosis multirresistente por Mycobacterium bovis e infección por el virus de la inmunodeficiencia humana. ¿Existen nuevas posibilidades terapéuticas? Enferm Infecc Microbiol Clin 2001; 19(1): 37-9.

15. Long R, Nobert E, Chomyc S, Van Embden J, McNamee C, Rey Durán R et al. Transcontinental Spread of Multidrug-resistant Mycobacterium bovis. Am J Respir Crit Care Med 1999; 159: 2014-7. 